\title{
Packet Filtering for Congestion Control under DoS Attacks
}

\author{
Yen-Hung $\mathrm{Hu}$ \\ Department of Computer Science \\ George Washingtin University \\ Washington, DC 20052 \\ yenhung@gwu.edu
}

\author{
Hongsik Choi \\ Department of Computer Science \\ Virginia Commonwealth University \\ Richmond, VA 23284 \\ hongsik@vcu.edu
}

\author{
Hyeong-Ah Choi \\ Department of Computer Science \\ George WAshington University \\ Washington, DC 20052 \\ hchoi@gwu.edu
}

\begin{abstract}
Congestion control in IP networks is typically done at each router through queue management, and the network is entirely dependent on the end hosts to react congestion. However, when misbehaving flows exist and continue to send their packets in very high rates, the queue management schemes implemented in current IP routers reveal a significant shortcoming in protecting legitimate flows. In this paper, we propose a novel scheme for congestion control in IP networks. Our approach is a time-window based filtering mechanism implemented in a router and processed before a queue management policy is applied. Setting the window size properly and dropping packets reaching in the next window can catch the non-responsive nature of misbehaving flows. The performance of our proposed scheme is demonstrated through extensive simulations using the NS2 simulator using a set of simulated traffic generated based on IP traces reported in http://www.nlnar.org.
\end{abstract}

\section{Introduction}

Congestion control in current IP networks is typically done at each router through queue management and packet scheduling policy. In this policy, the network is entirely dependent on the end hosts to react congestion, and it is expected that the flows will reduce their rates after packets are dropped (these are called responsive flows). The problem with this expectation is that misbehaving flows that do not cut down their sending rates after their packets are dropped will hog the buffer space at routers and deprive all other flows of their fair share of bandwidth (these are called unresponsive flows). An easy, yet very disruptive, way to cause unfairness to normal traffic is to congest the target links by sending high rate unresponsive flows, i.e., floodingbased denial-of-service (DoS) attacks. The network congestion created by such malicious flows cause most of the legitimate packets dropped at routers without reaching to their destinations.

The first-in-first-out (FIFO) queuing with drop tail policy is the simplest queue management policy for congestion control applied in most routers in the present Internet in which as long as the memory has open space, incoming packets are stored. The Random Early Detection (RED) is the most popular active queue management scheme in which the drop policy is dynamically changed in response to network traffic condition. These schemes work well when the flows are from properly implemented TCP. Several alternatives have been proposed to improve the situation with the objective of allocating fair share of bandwidth to each flow [2] [3] [10].

As it will be shown in the next section, the queue management schemes discussed above reveal significant shortcomings in protecting packets from normal flows when misbehaving malicious flows exist. In this paper, we propose a new approach to the control of network congestion when misbehaving malicious flows coexist with other normal flows. Our approach is time-window based filtering mechanism which is implemented in a router and processed before a queue management policy is applied. Setting the window size properly based on inter-arrival times for the high-rate packets and dropping packets reaching in the next window can catch the non-responsive nature of malicious flows.

The rest of this paper is organized as follows. In Sec- 
tion 2, shortcomings of existing queue managements and scheduling policies are discussed in the context of bandwidth fairness when unresponsive malicious flows coexist in the network. Our proposed scheme along with its technical rationale and its implementation issues are discussed in Section 3. Experimental results using the NS2 simulator are presented in Section 4. Section 5 concludes the paper.

\section{Queue Management Algorithms and Influ- ence of Misbehaving Unresponsive Flows}

In this section, we consider some of the queue management algorithms available in the literature and evaluate their performances in terms of their robustness to misbehaving malicious traffic flows. We identify five well-known algorithms for this study, and they are briefly described below.

- Drop Tail: This is the simplest policy on whether an incoming packet will be dropped or not. Any packet can be stored anywhere in the queue memory. As long as the memory has open space, incoming packets are stored. If any incoming packet finds the queue full, the packet is then dropped. This policy is currently used in the Internet.

- Random Early Detection (RED):[14] The gateway (router) detects incipient congestion by computing the average queue size and by dropping packets arriving at the gateway or by setting a bit in the packet headers. When the average queue size exceeds a threshold, the gateway drops or marks each arriving packet with a certain probability, where the exact probability is a function of the average queue size. RED gateways are designed to accompany a transport-layer congestion control protocol such as TCP. This scheme may work well when the flows are from properly implemented TCP, but it is possible for the buffer to overrun when the flows are non-responsive (i.e., flows continue to send packets at a very high rate without heed to the dropped packets).

- Stabilized RED (SRED):[17] This algorithm derived from the RED determines the drop probability of an incoming packet based on the hit frequency of the packet (by counting the number of packets arriving from the same flow during a certain time interval) in addition to the average queue size. Although the SRED discards packets with a load-dependent probability, the inheritance of the RED's limitation restricts its capability against high-rate unresponsive flows.

- Flow RED (FRED):[10] This algorithm is an extension of the RED using per-active-flow accounting in which a loss rate of each flow is maintained. The FRED only

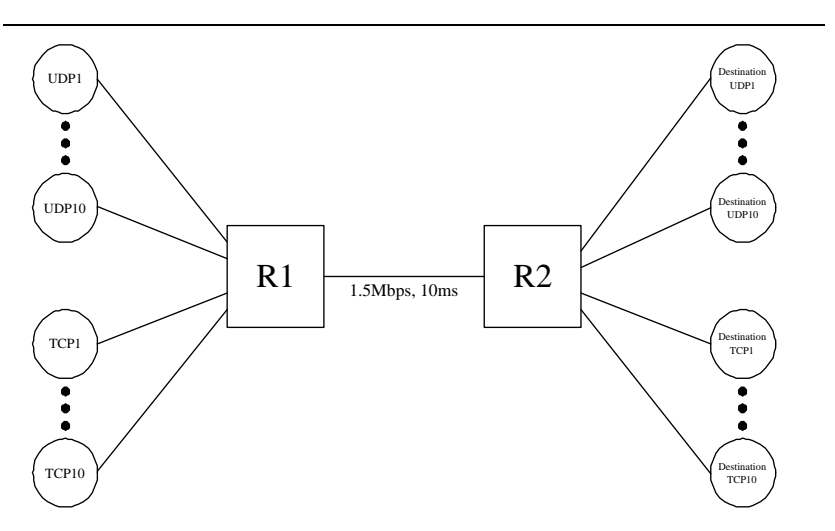

Figure 1. Network Topology

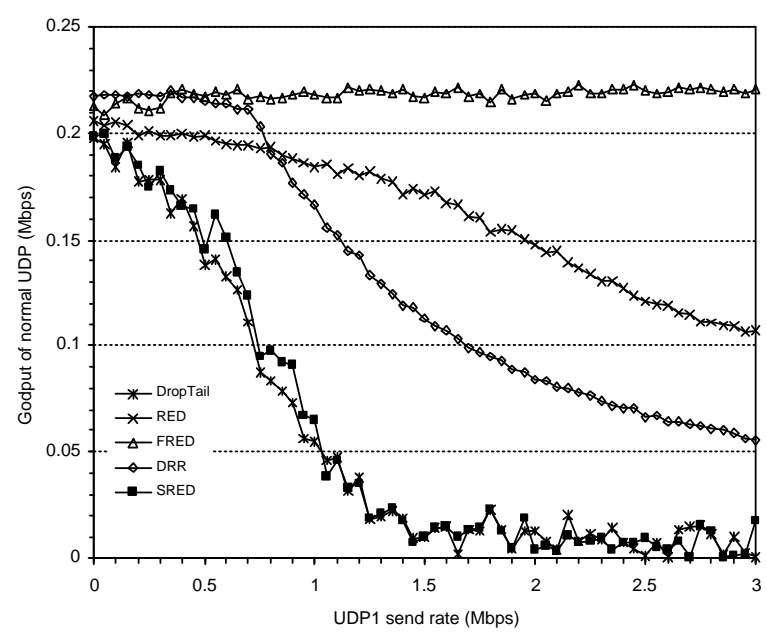

Figure 2. Goodput of Aggregate Traffic from UDP2-UDP10 vs. Sending Rate of UDP1.

keeps states of flows that have packets buffered in the gateway. The cost of the proposed per-active-flow accounting is is independent of the total flows traversing an Internet gateway, but it can be proportional to the buffer size.

- Deficit Round Robin (DRR):[11] This is a well-known weighted fair queuing discipline. It provides each flow approximated fairness by dropping packets from the longest per-flow queue. The DRR can provides better protection of bandwidth against high-rate unresponsive flows than the other four. But it also has limitation similar to SRED or FRED. 


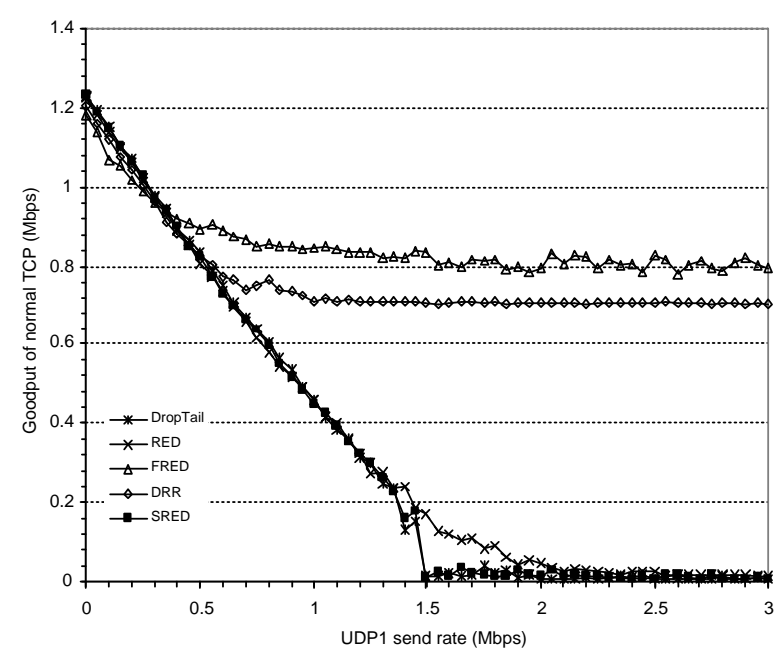

Figure 3. Goodput of Aggregate Traffic from TCP1-TCP10 vs. Sending Rate of UDP1.

We have implemented the above five algorithms using the NS-2 simulator for a simple network topology shown in Figure 1 in which 10 UDP flows (labeled UDP1 - UDP10) and 10 TCP flows (labeled TCP1 - TCP10) are competing for the bandwidth on the $1.5 \mathrm{Mbps}$ link between $R 1$ and $R 2$. Each of the 9 UDP flows UDP2 - UDP10 considered as normal flows has a constant sending rate $0.025 \mathrm{Mbps}$ (totaling $0.225 \mathrm{Mbps}, 15 \%$ of the $1.5 \mathrm{Mbps}$ bottlneck bandwidth), the sending rate of UDP1 varies from 0 to $3 \mathrm{Mbps}$ in each run of simulations. When the normal UDP flows (i.e., UDP2 - UDP10) are concerned, the FRED performs very well, providing almost full requested bandwidth. (See Figure 2.) However, the main drawback of the FRED is that it requires per-active-flow accounting, hence implementing the FRED may be infeasible when a large number of active flows exist. For example, [16] reports that 250,000 active flows are seen in an MCI router and 256,000 active flows are seen in an OC3 router in average. When the TCP flows are concerned, the fairness gets even worse as none of the algorithms including the FRED provides an acceptable performance. (See Figure3.)

The goodput of a flow is defined throughout this paper to be the ratio of packets reached to the destination over the total number of packets sent from the source of the flow.

\section{Window-Based Packet Filtering}

In this section, we discuss our new approach to congestion control that drops packets from high-rate unresponsive traffic streams. This filtering approach is based on a slid- ing time-window to identify the malicious nature of traffic streams, where the window size dynamically varies over time periods. Setting the window size based on the interarrival time for the packets from the suspected malicious flows and dropping packets reaching in the next window can catch the non-responsive nature of the malicious flows.

\subsection{Overview}

The initial assumption is that malicious flows tend to display a UDP-like traffic behavior and maintain a high flow rate without altering the flow identification (i.e., the source and destination addresses and port numbers) during the attack. On the other hand, legitimate flows such as TCP traffic are bursty in nature, and as a result there are periods of high and low activities. By carefully defining the window size, each burst is accepted during a single window and the low activity period is accommodated in the next window, resulting in higher throughput for normal TCP traffic. The high-rate UDP traffic on the other hand continues to send packets at constant rate and is thus active in every window.

Our proposed scheme is called the Window-Based Packet Filtering (WBPF), and the main idea is characterized as follows.

- The WBPF is turned on only if the aggregated arriving flow rate is larger than the available bandwidth of the output link and the queue experiences packet drops, i.e., the WFPF is on duing the congestion period.

- When the WBPF is on, a new incoming packet is dropped if a packet from the same flow has arrived in the previous consecutive window since it should be from a high rate flow.

- The time-window size is dynamically calculated based on several parameters observed during the current window period. A basic idea is that the next window size is increased if too many packet are still being dropped at the queue (i.e., not enough packets are filtered at the filter) and is decreased if the link utilization is too little while packets are being dropped at the filter (i.e., too many packet are filtered).

\subsection{Estimating Flow Rates}

Estimating the rate of flow $f$ was studied in $[5,8]$. The result in Equation (1) [5] is based on the time sliding window in which the rate $r_{f}^{\text {new }}$ of flow $f$ is updated from the corresponding old value $r_{f}^{\text {old }}$, packet inter-arrival time $\left.T_{f}^{k}=t_{f}^{k}-t_{f}^{k-1}\right)$, the size of arriving packet $\left(l_{f}^{k}\right)$, and a constant $\kappa$.

$$
r_{f}^{\text {new }}=\frac{r_{f}^{o l d} \times \kappa+l_{f}^{k}}{T_{f}^{k}+\kappa}
$$


In the second model shown in Equation (2), the exponential averaging was used to estimate the flow rate:

$$
r_{f}^{n e w}=\left(1-e^{\frac{-T_{f}^{k}}{\kappa}}\right) \frac{l_{f}^{k}}{T_{f}^{k}}+e^{\frac{-T_{f}^{k}}{\kappa}} r_{f}^{o l d}
$$

The results in Eq.( 1) and Eq.( 2) can be modified to estimate the aggregate flow rate by:

$$
R^{\text {new }}=\frac{R^{\text {old }} \times \kappa+l^{k}}{T^{k}+\kappa},
$$

and

$$
R^{n e w}=\left(1-e^{\frac{-T^{k}}{\kappa}}\right) \frac{l^{k}}{T^{k}}+e^{\frac{-T^{k}}{\kappa}} R^{o l d},
$$

where $R^{\text {new }}$ and $R^{\text {old }}$ denote the new and old values of the aggregate flow rates, $l^{k}$ denotes the length of the packet just arrived, and $T^{k}$ denotes the inter arrival time of two consecutive packets (without identifying flow ids).

During the simulation study, we have observed that Equations (3) and (4) produce similar results in terms of goodputs, and all results reported in Section 4 are based on Equation (4).

\subsection{Computing Time-Window Size}

Let $s(i)$ denote the size of the current window. In computing the next window size $s(i+1)$, we use three design parameters $\alpha, \beta$, and $\delta$. The values of $\alpha$ and $\beta$ are in $0<\alpha, \beta<1$. In every small period of $\delta$ milli-seconds, two actions are performed: (1) the queue status is updated such that $Q_{-} D(\delta)=1$ if and only if at any time instance, at least one packet was dropped within $\delta$ milli-seconds and (2) the average packet size packet_size $e_{a v e}(\delta)$ is calculated. (At any instance of time, packet_size ave $(\delta)$ denotes the most recent value of the average packet size, i.e., the value calculated within $\delta$ milli-seconds.) The optimal values of $\alpha, \beta$, and $\delta$ are to be determined based on experiments. The link utilization during the window period $i$ is denoted by $u(i)$. The drop rate at the queue during the window period $s(i)$ is defined as $R_{Q \_} D(i)=D(i) / B$, where $D(i)$ denotes the total number of bits dropped at the queue during window period $s(i)$ and $B$ (bits per seconds) denotes the link bandwidth.

When the filter is turned on, the initial window size is set to be $s(0)=$ packet_size $_{a v e}(\delta) /(\alpha B)$. The underlying idea of this set-up is that the initial window size is set to be very large such that packets from flows with arrival rates larger than $\alpha$ times the link bandwidth will only be dropped at the filter. The optimal value of $\alpha$ is expected to be smaller at routers in high-bandwidth networks and larger at routers in low-bandwidth networks.

Consider two cases at the end of current window period. If packets are still being dropped at the queue (i.e.,
$Q \_D(\delta)=1$ ), the next window size will be increased to drop more packets at the filter. Note that if window size is increased such that the amount of dropped packets at the filter is not larger than the amount of dropped packets at the queue when the window size is not increased, the overall link utilization is not decreased while possibly providing better fairness to low-rate flows. The increment is based on parameters observed during the current window period: $s(i)$, packet_size ${ }_{\text {ave }}(\delta)$, and $R_{Q \_D}(i)=D(i) / B$. More specifically, we set

$s(i+1)=s(i)+\beta * s(i) *$ packet_size $_{\text {ave }}(\delta) * R_{Q \_D}(i)$.

As a second case, suppose no packet is dropped at the queue (i.e., $Q \_D(\delta)=0$ ). If the link utilization $u(i)$ of the current period is less than $100 \%$, the next window size has to be decreased as too many packets were dropped at the filter during the current period. In this case, we set

$$
s(i+1)=s(i)-(1-u(i)) * s(i) .
$$

If no packet is dropped at the queue but the link is fully utilized (i.e., $u(i)=1$ ) during the current period, we keep the current window size for the next period, i.e.,

$$
s(i+1)=s(i)
$$

The detailed description of our WBPF algorithm is given in the next section.

\subsection{WBPF Algorithm}

The following additional notations will be used in the description of our algorithm. $F=1$ means that the filter is on. The set of flow identifications seen during the window period $i$ is denoted by $L(i)$. 


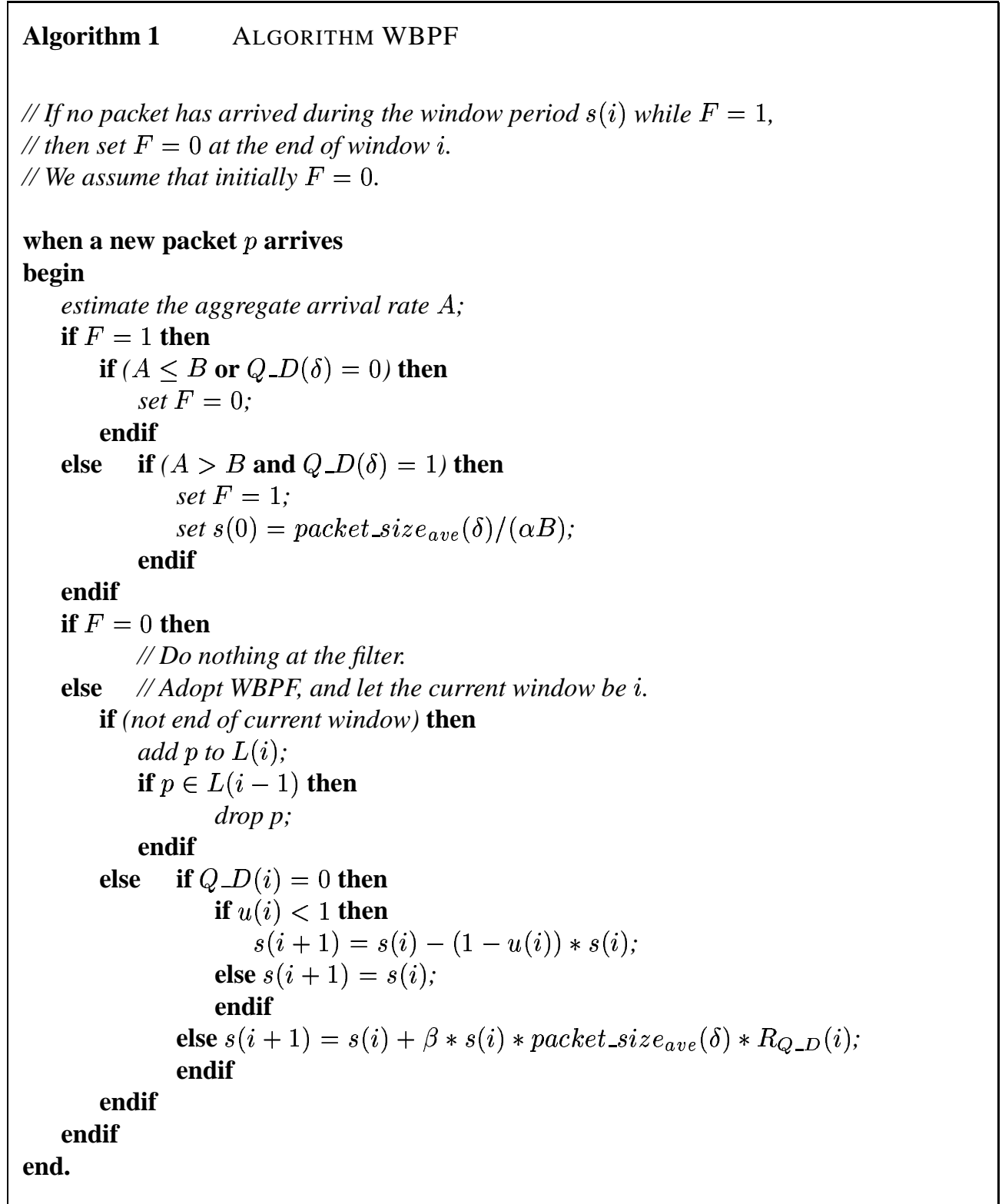

Figure 4. WBPF Algorithm 


\section{Experimental Results}

We have implemented the WBPF module in the NS-2 simulator and compared its performance against two queue management algorithms, the drop-tail and the RED. Two network topologies were considered in our simulations: a single link simulating the Merit Abilene link and the NSF T1 topology.

\subsection{System Parameters in Simulations}

Throughout the simulations, we set $\alpha=\beta=0.5$ and $\delta=5$ milli-seconds. The maximum buffer size of each router (in both topologies) is 40 packets. The mean propagation delay of each link is $10 \mathrm{~ms}$. A constant value of $\kappa$ used in Equations (1)-(4) to estimate flow rate is set as $40 \mathrm{~ms}$. We use the default values in [14] and [10] for RED and FRED, where $m i n \_t h, m_{a x} \_t h, q \_w e i g h t, m a x \_p$ are 5, 15, 0.002, 0.1 , respectively.

\subsection{Simulation based on IP Traces Collected at Merit Abilene}

We have obtained IP traces (MRA-1060983892-1.tsh.gz marked on 15 Aug. 2003 15:01) from the NLANR (http://www.nlanr.org). The traffic was monitored and collected from an OC-12 line (622Mbps) during a $90 \mathrm{sec}-$ ond period at Merit Abilene site. The analysis of collected data shows that the total number of packets is $1,375,457$ with the average packet size about 600 bytes. The total amount of traffic is $937,360,736$ bytes of which about $88 \%$ are from TCP flows and $12 \%$ from UDP flows.

We have calculated the Hurst parameter $H$ (see ?? for the definition of and an algorithm to compute $H$ ) of three different groups: packets from TCP flows, packets from UDP flows, and overall packets. All three groups show the $H$ value larger than 0.5 , hence exhibiting a high degree selfsimilarity. The Hurst parameter will be discussed further in the next section when we discuss on the simulated traffic denoting aggregate malicious unresposnive flows.

A summary of our analysis of the MRA traffic is given in Table 1.

4.2.1. Performance Analysis in Normal Condition In order to test the false positive rate of our WBPF algorithm under a normal network condition, we have closely examined the MRA traffic. There are 22,038 flows (flows are identified using source addresses only), and we have identified 319 flows which make up 872,239,014 bytes, about $93 \%$ of the overall bytes. Due to the computational complexity, we assumed 320 flows (319 large flows and others all considered as a single flow) in our simulations.

As shown in Figure 5, the utilization of the MRA Abilene link (622Mbps) is all-time less than $16 \%$ without expe-

\begin{tabular}{|c|c|c|c|}
\hline & TCP & UDP & Overall \\
\hline \hline \# of Packets & $1,375,457$ & 182,273 & $1,568,142$ \\
\hline \% of Packets & 87.71 & 11.62 & 100 \\
\hline \# of Bytes & $803,478,195$ & $133,085,187$ & $937,360,736$ \\
\hline \% of Bytes & 85.71 & 14.19 & 100 \\
\hline Ave Packet Size & 584.15 & 730.14 & 597.75 \\
\hline Hurst Parameter & 0.790 & 0.637 & 0.786 \\
\hline
\end{tabular}

Table 1. Summary of MRA Traffic Analysis

riencing any packet drop. So we have reduced the link bandwidth from 644Mbps to 77Mbps and performed simulations using the identical traffic. Figure 6 shows that the link utilization is still less than $100 \%$, where we were only able to compute link utilization in every 0.5 second due to the computational limit. However, packets can be still dropped during burst periods (see Figure 7) even if the link is under utilized over a longer period.

As the WBPF algorithm is potentially making additional packet drops at the filter, one may expect that more packets may be dropped when the WBFP is on under a normal network condition. However, it is observed that the performance of our WBPF algorithm is similar to other algorithms. In fact, it is better than the RED. Hence, its false positive rate is shown to be almost negligible.

4.2.2. Performance Analysis in the Presence of Malicious Flows Now, we are introducing packets from highrate unresponsive flows. Such flows may be coming from multiple sources and destined to multiple destinations. As no clear characteristics on such flows has been studied in the literature, we use the Hurst parameter of the aggregate traffic from such flows when monitored at a single point as an input variable in generating simulated malicious packets. Note that we do not differentiate flow ids from those packets, and we simply consider the $\mathrm{H}$ value of the aggregate flows. A code published in http://ita.ee.lbl.gov/html/contrib was implemented in the NS2 simulator. This program takes the $\mathrm{H}$ value as an input and generate as an output a time series whose $\mathrm{H}$ value matches with the given input. For five different values of $H$, the simulated malicious traffic was combined with the MRA traffic and applied to the 77Mbps link. (Note that the rate of the aggregate combined traffic exceeds the link bandwidth 77Mbps.) Figure 8 - 12 show the performance of drop-tail, RED, and our WBPF algorithms in terms of link utilizations over the 90 second period. Note that labels $\mathrm{X}-\mathrm{N}$ and $\mathrm{X}-\mathrm{A}$ in the figures show the link utilization of packets from normal and attacking flows, resp., when algorithm $X$ is applied. In all cases, our WBPF algorithm performs significantly better than others. 


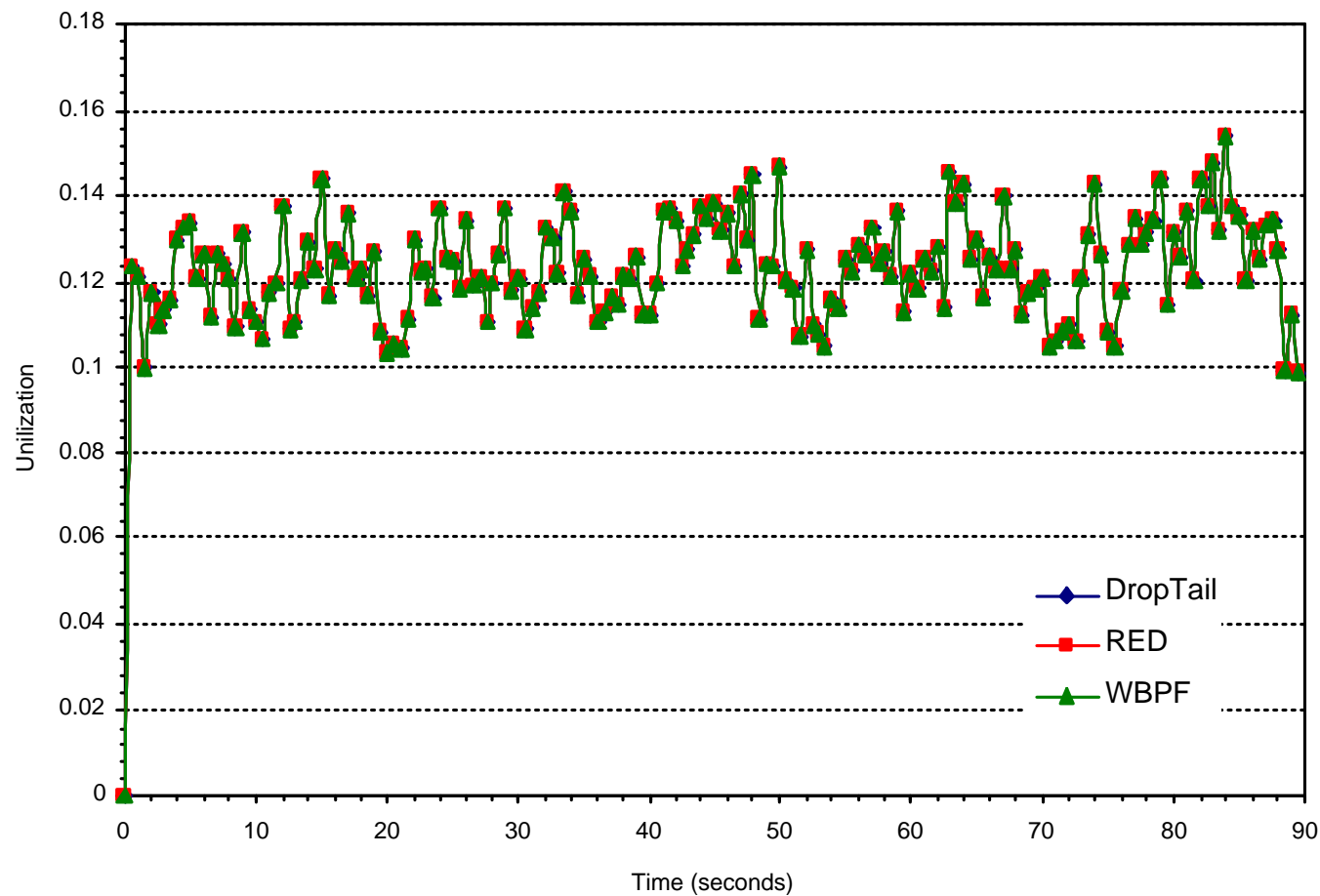

Figure 5. Utilization of $622 \mathrm{Mbps}$ Link 


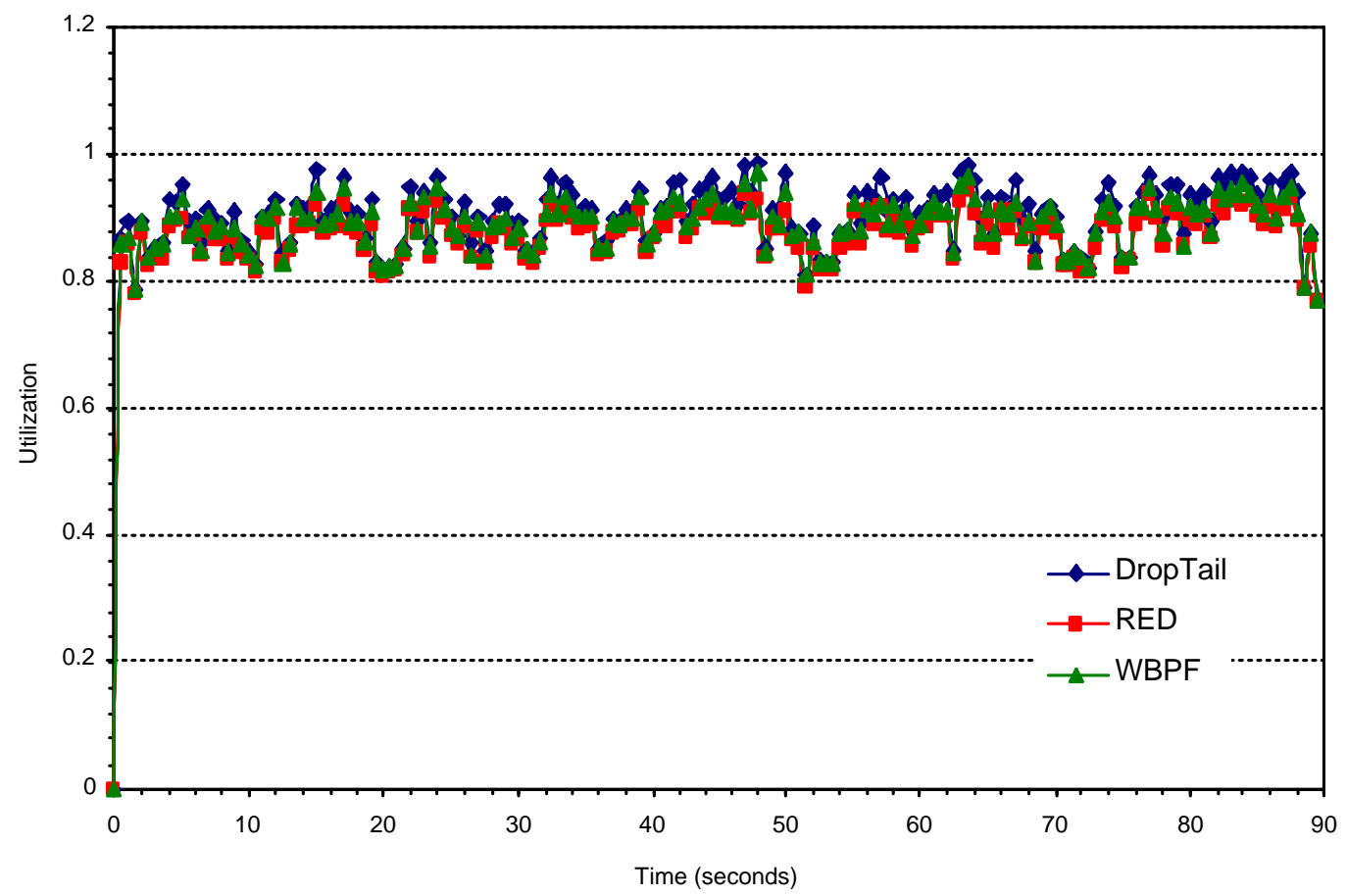

Figure 6. Utilization of $77 \mathrm{Mbps}$ Link 


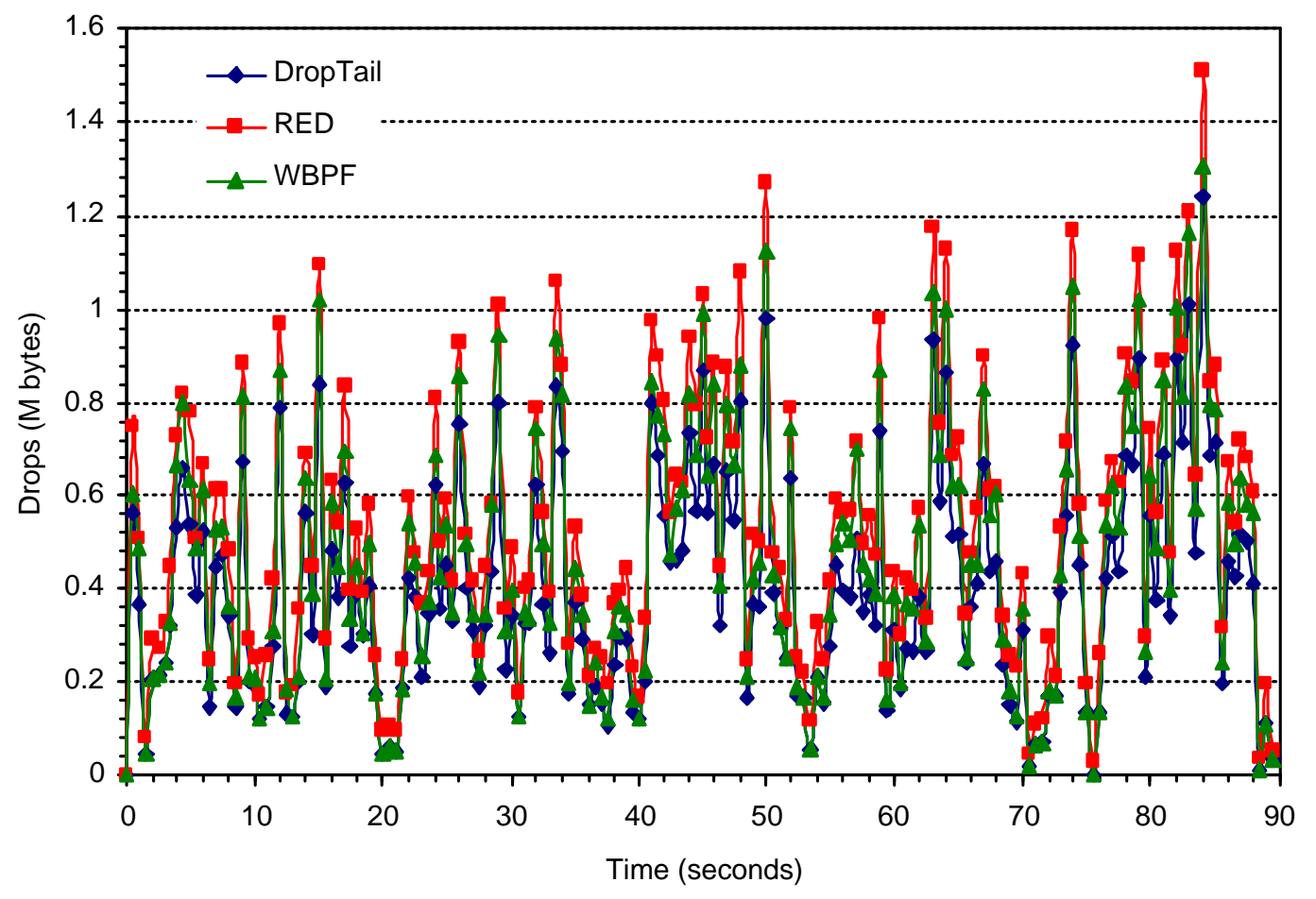

Figure 7. Packet Drops at $77 \mathrm{Mbps}$ Link 


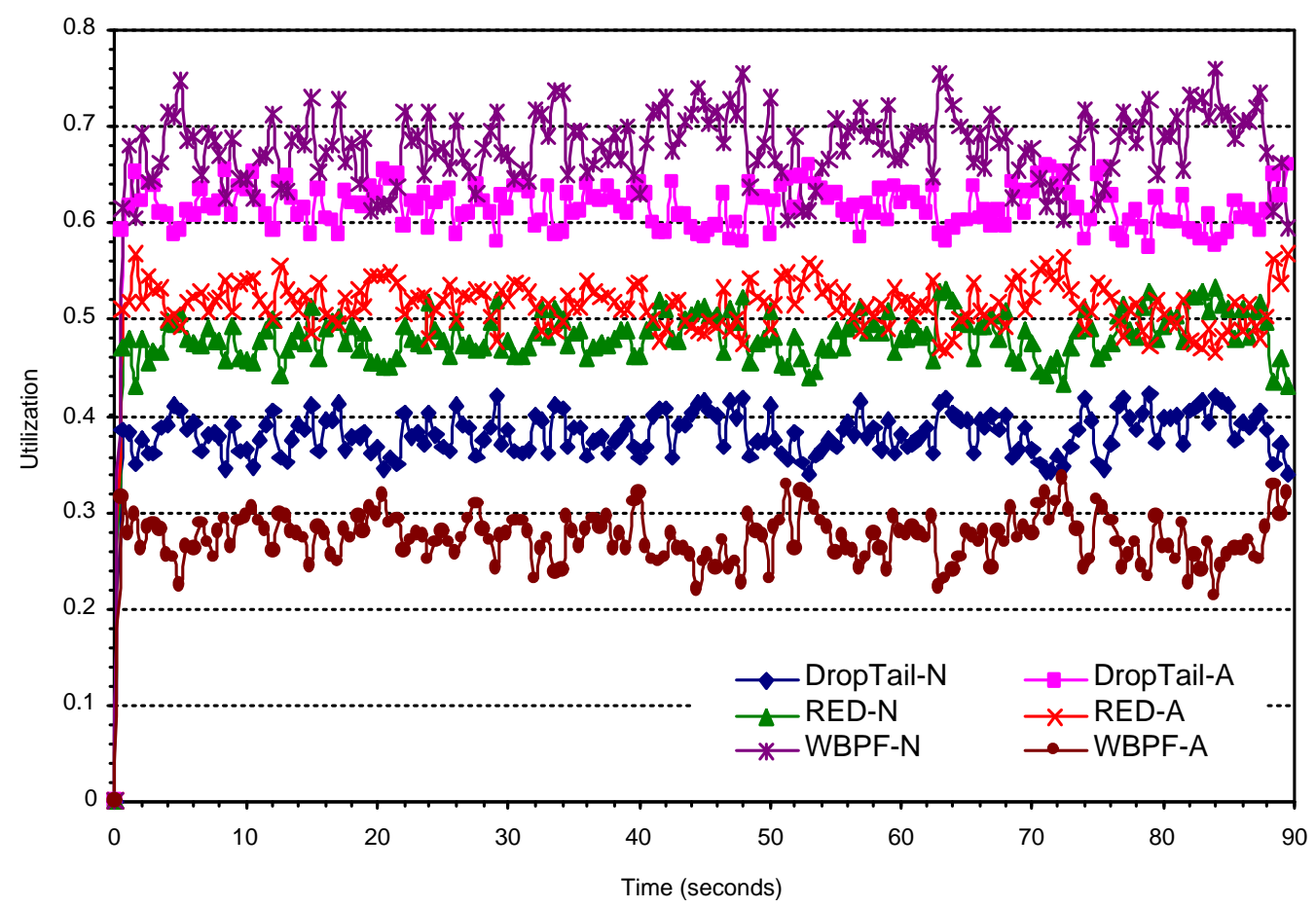

Figure 8. Malicious traffic with $\mathbf{H}=0.55$ 


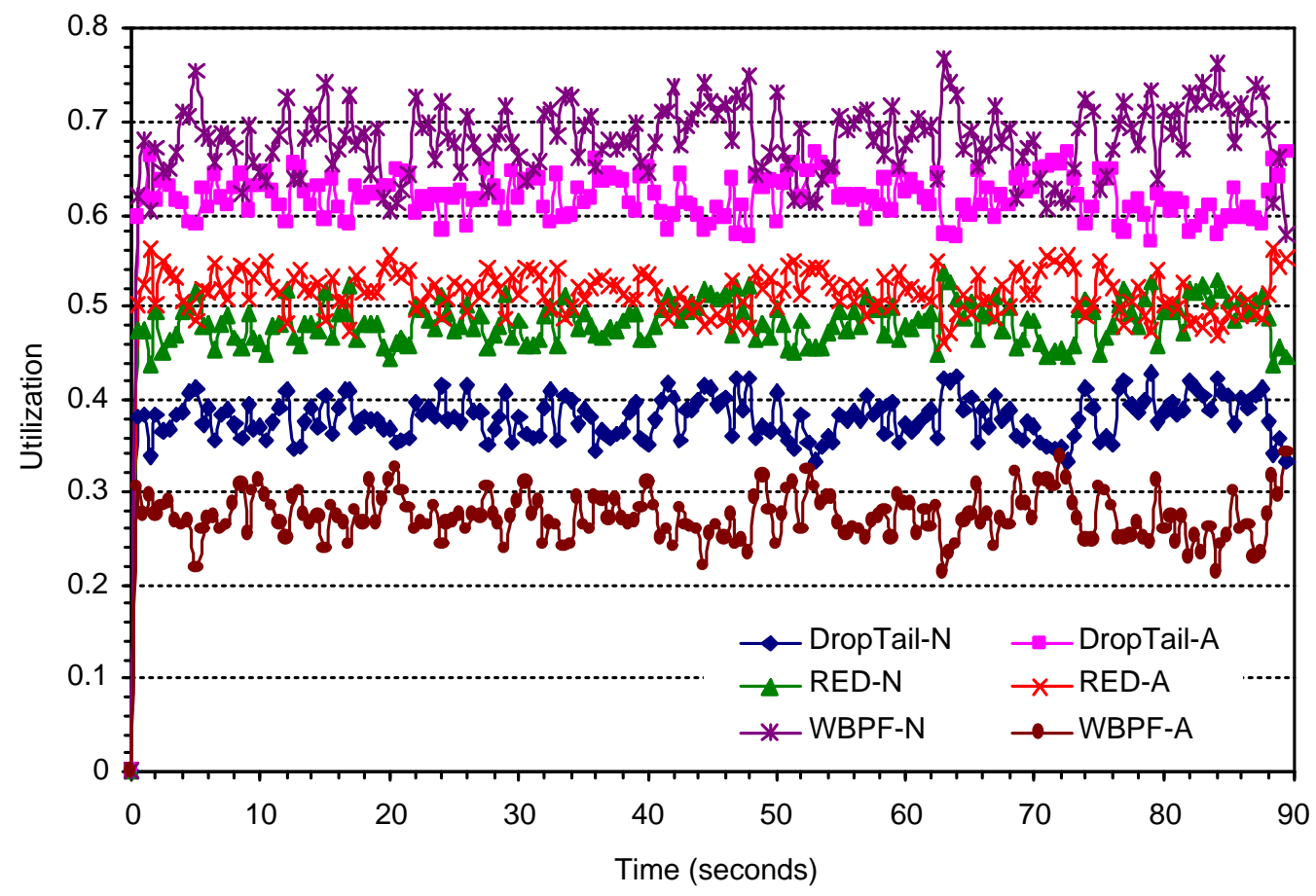

Figure 9. Malicious traffic with $\mathrm{H}=\mathbf{0 . 6 5}$ 


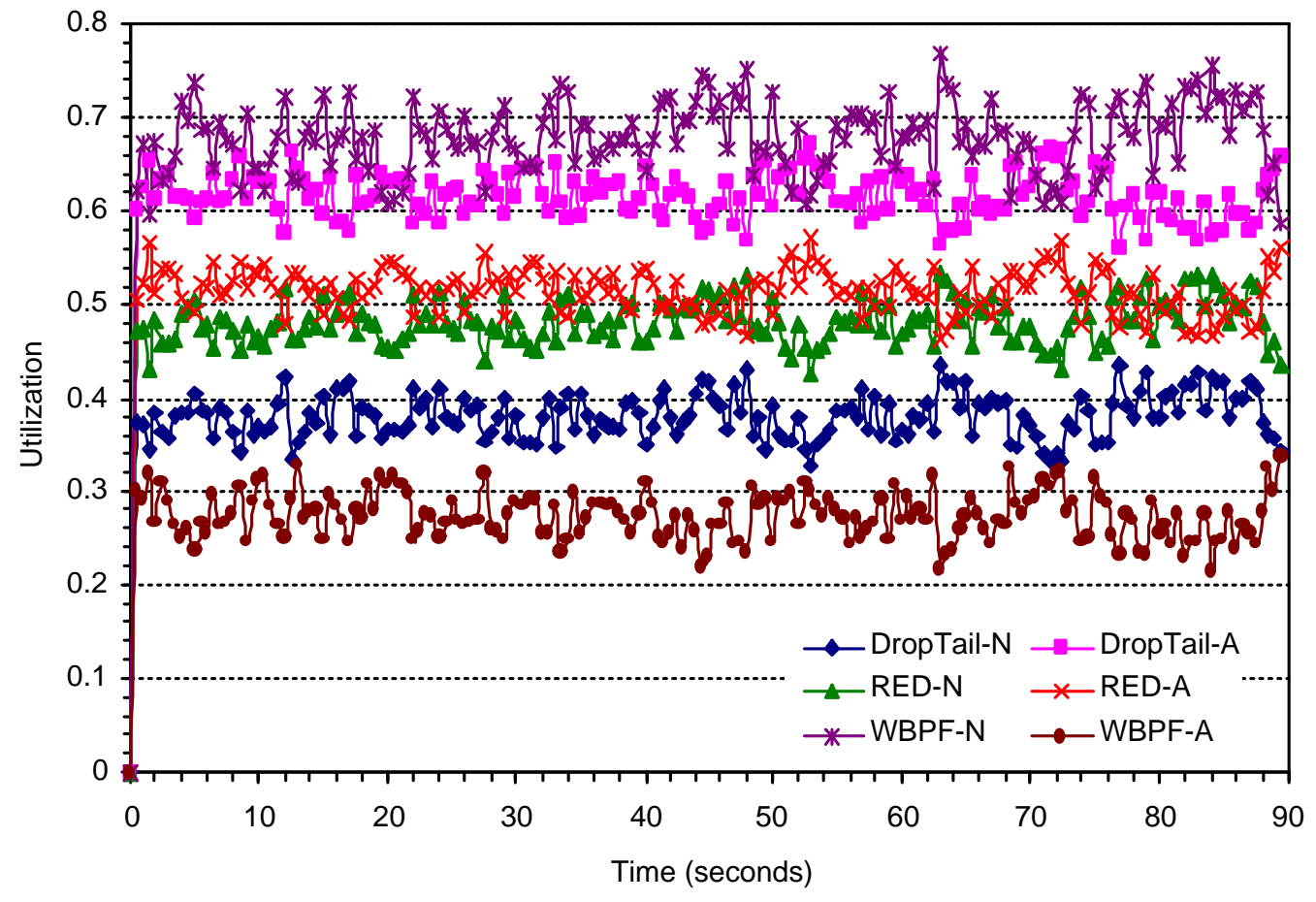

Figure 10. Malicious traffic with $\mathbf{H}=\mathbf{0 . 7 5}$ 


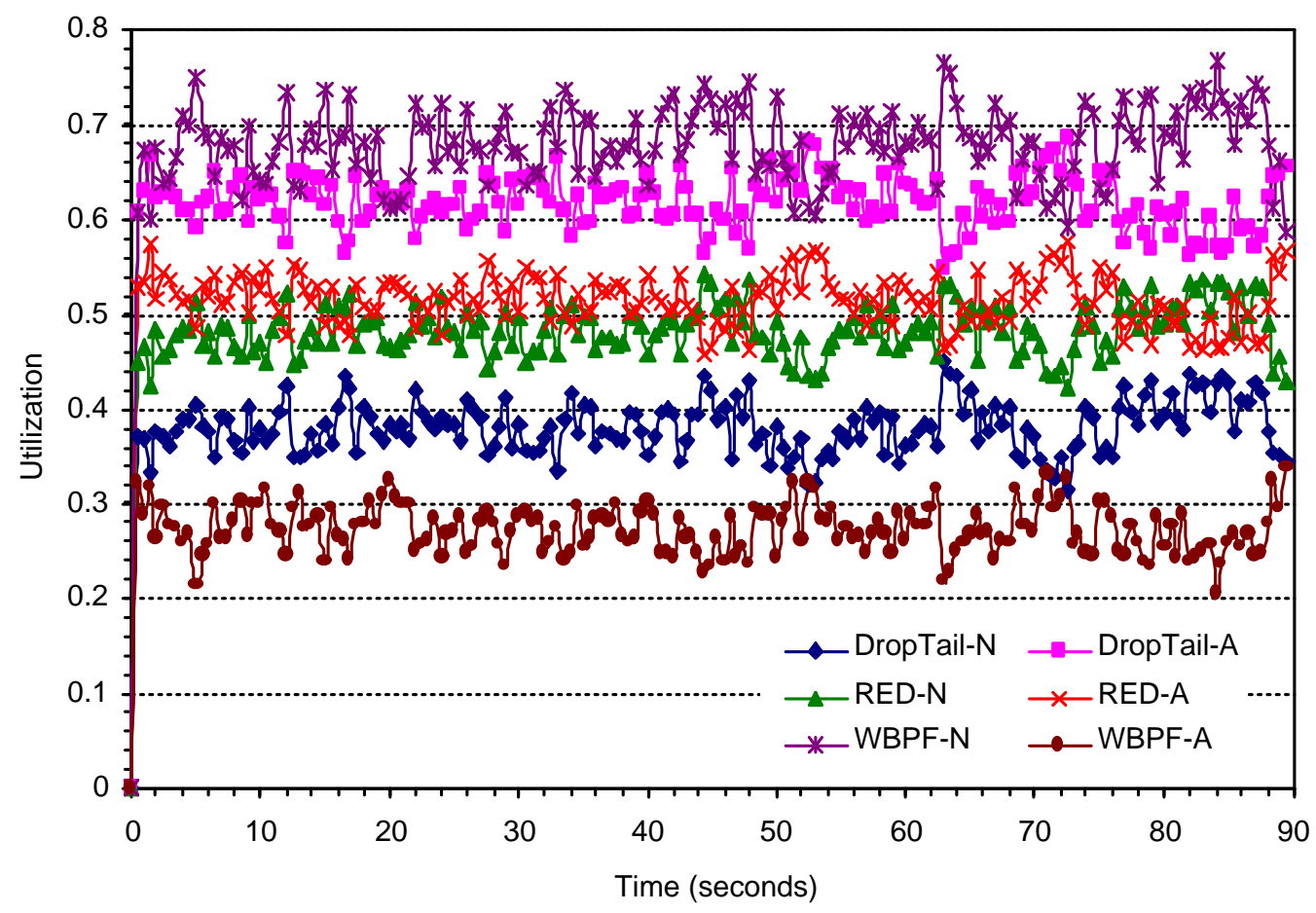

Figure 11. Malicious traffic with $\mathrm{H}=\mathbf{0 . 8 5}$ 


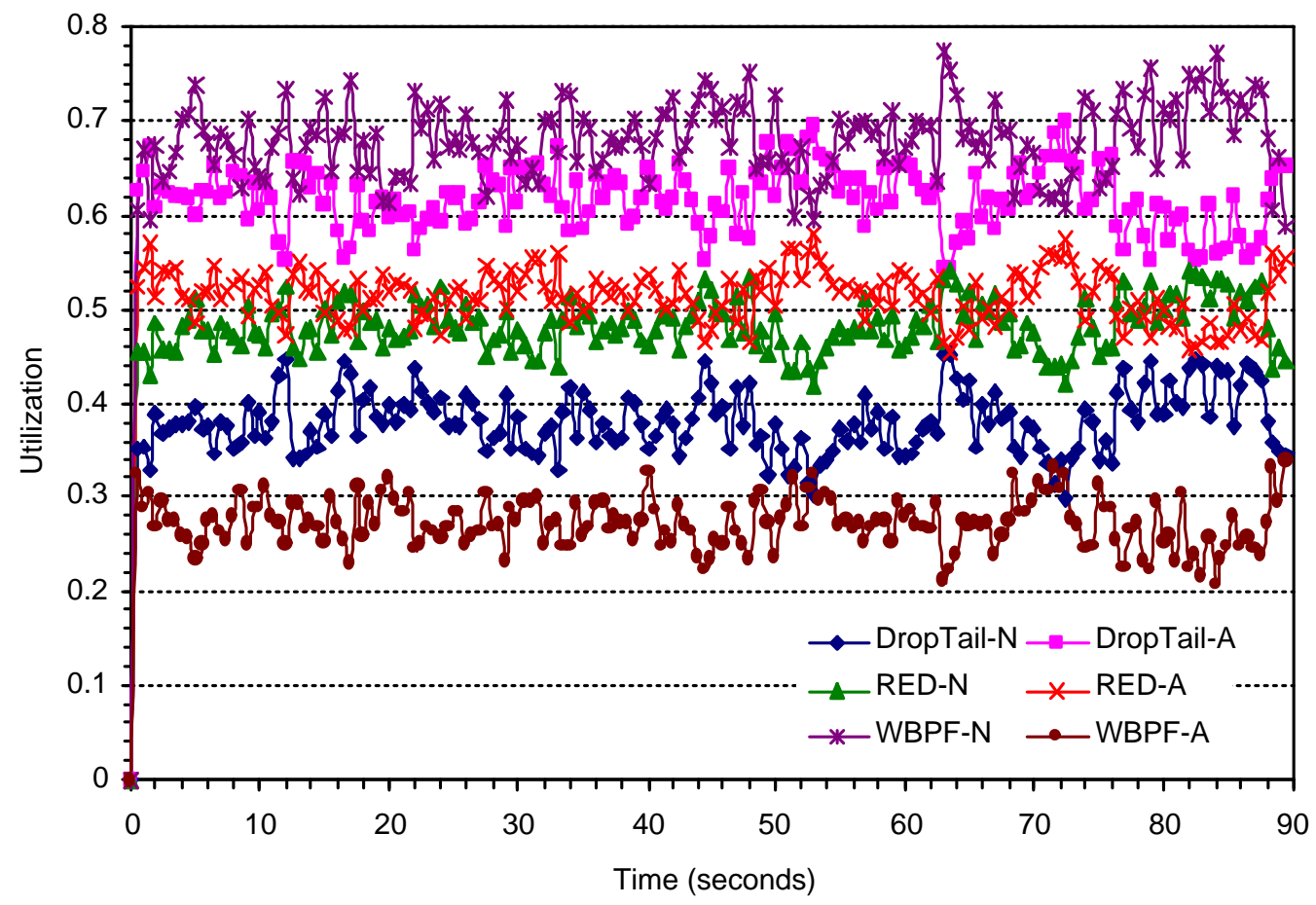

Figure 12. Malicious traffic with $\mathrm{H}=0.95$ 


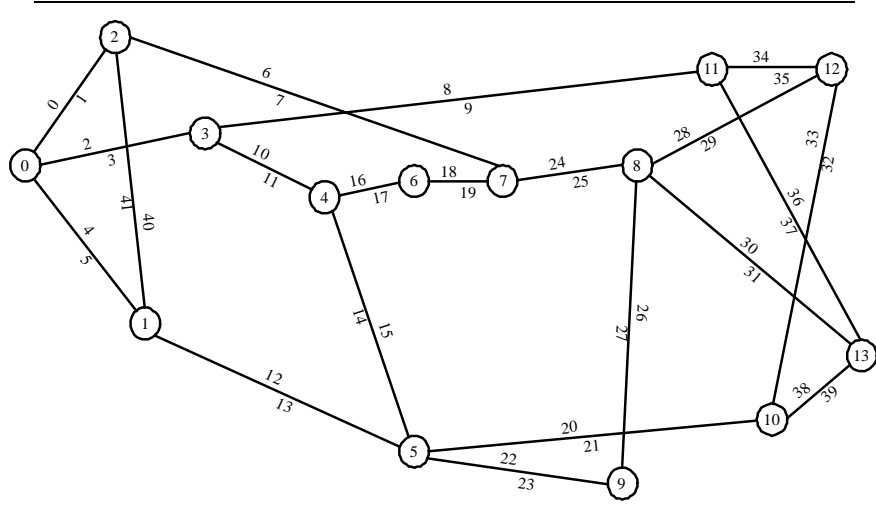

Figure 13. NSF T1 Topology.

\subsection{Simulation on NSF Topology}

In this experiment, we study how the goodput of normal traffics is affected when they traverse more than one congested links. Several experiments are performed on the NSF T1 topology (see Figure 13) where each link has bandwidth $1.544 \mathrm{Mbps}$ and propagation delay $10 \mathrm{~ms}$. We have deigned traffic pattern for this topology (see Figure 14) based on the real traffic report in [1]. Each type of traffic has a certain number of flows and traffic load. The path and the start time of each flow are carefully chosen to provide the overall average utilization of this network close to $39 \%$. The stop time of each flow is varied based on the flow load as well as the network conditions. In our setup, there are 223 UDP flows and 924 TCP flows traversing through the NSF T1 network. Among these 1147 flows, 252 of them pass 1 hop, 448 of them pass 2 hops, and 447 of them pass 3 hops, resulting in the average hop 2.17.

When one misbehaving flow enters the network affecting link 24 and link 28 (source and destination nodes are 7 and 12), our simulation result (see Figure 15) shows that only WBPF can efficiently drop packets from the misbehaving flow. The FRED and DRR allow some bandwidth to the misbehaving flow, but when the DropTail or RED is applied, the misbehaving flow fully dominates the two links 24 and 28.

We then perform additional experiments by increasing the number of misbehaving flows: $1-5$. The rate of these flows are all higher than the available bandwidth creating a severe network congestion without a proper action. Our results (see Figure 16 and 17) show that WBPF performs as well as most of the packets from the misbehaving flows are filtered out. However, other algorithms decrease their overall goodputs even further when the number of misbehaving flows increase.

\begin{tabular}{|c|c|c|c|}
\hline & Types & Flow Number & Packets / Flow \\
\hline \multirow{6}{*}{ ণ } & MULTICAST & 35 & 10456 \\
\hline & REAL & 6 & 1968 \\
\hline & HALF LIFE & 27 & 144 \\
\hline & ICQ & 4 & 112 \\
\hline & DNS & 53 & 8 \\
\hline & OTHERS & 98 & 96 \\
\hline \multirow{7}{*}{$\stackrel{-1}{0}$} & NNTP & 295 & 1880 \\
\hline & FTP & 15 & 1232 \\
\hline & QNUTELLA & 33 & 464 \\
\hline & NAPSTER & 10 & 1500 \\
\hline & HTTP & 383 & 32 \\
\hline & KAZAA & 14 & 472 \\
\hline & OTHERS & 174 & 384 \\
\hline
\end{tabular}

Figure 14. Traffic for NSF T1 Topology.

\begin{tabular}{|r|r|r|r|r|r|}
\hline & & & & \\
\hline Attacker ratio of BW & DropTail & RED & FRED & DRR & WBFA \\
\hline Link 24 & 0.97 & 0.88 & 0.24 & 0.55 & 0.00 \\
\hline Link 28 & 0.93 & 0.77 & 0.21 & 0.48 & 0.00 \\
\hline
\end{tabular}

Figure 15. Ratio of Bandwidth Occupied by Misbehaving Flow.

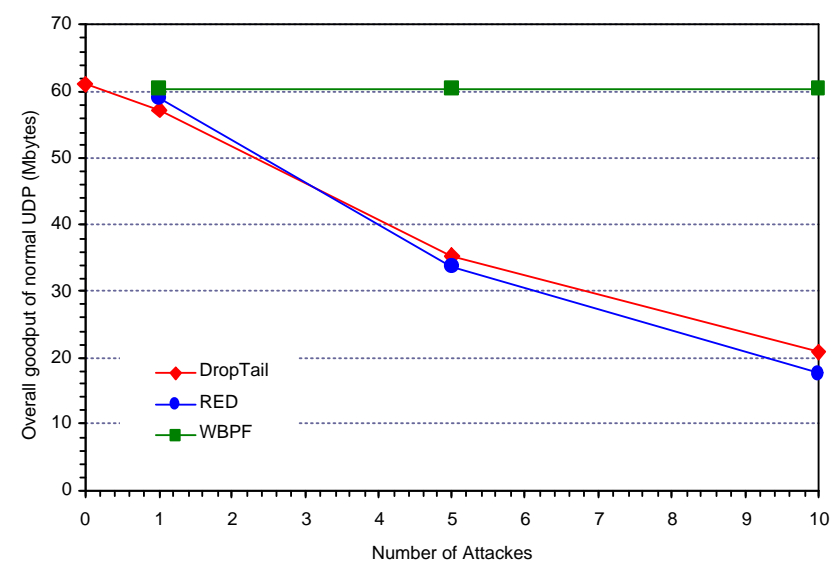

Figure 16. Aggregate Goodput of Normal UDP Flows vs. Number of Malicious Flows. 


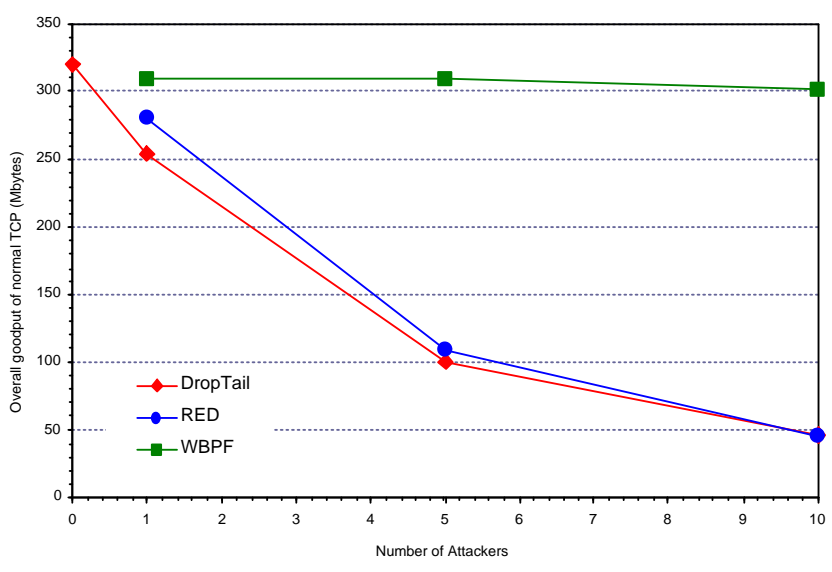

Figure 17. Aggregate Goodput of TCP Flows vs. Number of Malicious Flows.

\section{Conclusions and Future Work}

In this paper, we have proposed a time-window based packet filtering (WBPF) approach to controlling network congestion caused by misbehaving unresponsive flows. Our approach catches the unresponsive nature of misbehaving unresponsive flows. Technical rationale of our approach and a concrete description of our algorithm were presented. Through the simulation study, it was verified that our WBPF performs very well and fully controls misbehaving unresponsive flows while other existing queue management policies fail to provide protection of legitimate flows.

However, to deploy our scheme in real network environments, many research issues further remain. Firstly, it is essential to develop an implementation technique (hardware or software) to efficiently maintain the lists used in the WBFA. Secondly, the proposed WBFA can only detect and control high rate unresponsive flows at local routers. However, intelligent attackers would minimize the nonresponsive nature of individual flows. It is then necessary to coordinate the various flow monitors to identify collaborating flows.

\section{Acknowledgement}

This work was supported in part by the DARPA under Grant N66001-01-1-8932 (co-funded by NSA).

\section{References}

[1] J. St .Sauver, "Oregon Gigapop traffi c characterization," Internet2/NLNAR Joint Techs May 16th, 2001, Lincoln NE.
[2] A. Demers, S. Keshav, and S. Shenkar, "Analysis and simulation of a fair queueing algorithm," J. Internetw. Res. Experience, pp. 3-26, Oct. 1990.

[3] A. K. Parekh and R. G. Gallager, "A generalized processor sharing approach to fbw control in integrated services network: The single-node case," IEEE/ACM Trans. on Networking, vol. 1, No. 3, June, 1993.

[4] D. Lin and R. Morris, "Dynamics of random early detection," Proc. ACM SIGCOMM, Cannes, France, Oct. 1997, pp. 127137.

[5] D. D. Clark and W. Fang, "Expliciit ALlocation of Best Effort Packet Delivery Service," IEEE/ACM Trans. Networking, vol. 6, no. 4, AUgust 1998.

[6] S. Floyd and K. Fall, "Promoting the use of end-to-end congestion control in the internet," IEEE/ACM Trans. Networking, vol. 7, pp. 458-472.

[7] NLANR PMA, Aiblence-I data set, "ttp://pma.nlanr.net/Traces/long/ipls1.html"

[8] I. Stoica, S. Shenker, and H. Zhang, "Core-stateless fair queueing: a scalable architecture to approximate fair bandwidth allocations in high-speed networks," IEEE/ACM Trans. Networking, vol. 11, no. 1, Feb. 2003.

[9] I. Stoica, S. Shenker and H. Zhang, "Core-stateless fair queueing achieving approximately fair bandwidth allocations in high speed network," Carnegie Mellon Univ., Pittsburgh, PA, Tech. Rep. CMU-CS-98-136, 1998.

[10] D. Lin and R. Morris, "Dynamics of random early detection," in Proc. of SIGCOMM '97.

[11] M. Shreedhar and G. Varghese, "Effi cient fair queueing using defi cit round robin," in Proc. of SIGCOMM '95, Cambridge, MA, USA.

[12] The network simulator NS2, "http:www.isi.edunsnamns".

[13] S. McCreary, K. Claffy, "Trends in wide ared IP traffi c patterns," ITC Specialist Seminar, Monterey, CA, Sep. 18-20, 2000.

[14] S. Floyd and V. Jacobson, "Random early detection gateways for congestion avoidance," IEEE/ACM Trans. on Networking, 1(4), Aug. 1997.

[15] P. Karn and C. Partridge, "Improving Round Trip Time Estimates in Reliable Transport Protocols," ACM SIGCOM'87, pp. 2-7, August 1987.

[16] K. Tomson, G.J. Miller, and R. Wilder, "Wide area traffi c patterns and characteristics," IEEE Networks, Dec. 1997.

[17] T. J. Ott, T. V. Lakshman, and L. Wong, "SRED: Stabilized RED," in Proceedings of IEEE INFOCOM99, Mar. 1999.

[18] Will E. Leland, Murad S. Taqqu, Walter Willinger, and Daniel V. Wilson, "On the Self-Similar Nature of Ethernet Traffi c (Extended Version)," IEEE/ACM Transactions on Networking, Vol. 2, No. 1, February 1994. 